\title{
Servicios de intermediación laboral: enseñanzas para América Latina y el Caribe
}

\section{Jacqueline Mazza \\ Especialista Senior en Desarrollo Social Banco Interamericano de Desarrollo (BID) jaquelinem@iadb.org}

En una época de cambios laborales acelerados, se otorga cada vez más importancia a los sistemas de intermediación laboral como herramienta capaz de mejorar la transparencia y el funcionamiento de los mercados laborales. En este artículo se identifican seis modelos de sistemas de intermediación y se concluye que, dados los recursos limitados y la estructura institucional de América Latina y el Caribe, los modelos C, de asociación entre el sector público y el privado, y F, de servicios basados en el sector privado, son los que más se adecuan a las economías de la región. Para que los sistemas de intermediación laboral se orienten más a satisfacer las necesidades de la región se deberán adaptar a las circunstancias nacionales, como la existencia de grandes sectores informales, migración interna y externa, sistemas de capacitación ineficaces y mecanismos inadecuados de formulación de políticas para el mercado laboral. Gracias a adaptaciones en el diseño de los sistemas, los renovados servicios de intermediación laboral permitirán abordar problemas urgentes del mercado de trabajo, como la alta rotación laboral, la discriminación, y la falta de coordinación entre el empleo y la capacitación de los recursos humanos. 
I

\section{Introducción}

En todos los países existen brechas y desfases entre la demanda de trabajadores por parte de los empleadores y la oferta de interesados en conseguir trabajo. La ausencia de un mercado de trabajo de eficiencia plena (fully clearing) es producto de numerosos factores, como la escasa y poco difundida información sobre las oportunidades laborales, los desencuentros entre la calificación que tienen los trabajadores y la que requieren los empleadores, la habilidad en general insuficiente de las personas para buscar empleo y la discriminación en el mercado laboral. Estas brechas y desfases entre la oferta y la demanda pueden tener importantes consecuencias económicas que se manifiestan en aumento y prolongación del desempleo y el subempleo y en deterioro de la productividad. Los progresos de la integración económica han elevado el ritmo y las exigencias de adaptación a tasas más altas de rotación entre puestos de trabajo, cambios de carrera y capacitaciones más frecuentes para aumentar las destrezas laborales.

La evolución del mercado laboral en América Latina y el Caribe sólo recientemente ha hecho patentes las graves dificultades para integrar a los trabajadores, tal como se aspiraba, a un ciclo permanente de educación, trabajo y capacitación. Las tendencias recientes en la región son: la tasa de desempleo se ha elevado, sobre todo en el Cono Sur y la zona andina; la rotación entre puestos de trabajo va en aumento en muchos países; el subempleo es alto, sobre todo en Centroamérica, y la región depende considerablemente de métodos ineficientes e informales de colocación.

Los servicios públicos nacionales de empleo, creados en las economías industriales de principios del siglo XX, están siendo reestructurados en muchos países con el fin de adaptarlos a las nuevas exigencias derivadas de cambios de empleo más frecuentes. Para referirse a los sistemas resultantes de esta reformulación de las políticas de los servicios nacionales de empleo resulta más adecuado llamarlos servicios de intermediación laboral, ya que sus prestaciones se han ampliado para abarcar la "intermediación" entre el trabajador, el empleo, la educación y la capacitación, el trabajo por cuenta propia y otros servicios sociales necesarios. Los servicios de intermediación laboral son hoy una herramienta de política para mejorar la calidad y la eficiencia en el proceso de cuadrar a quienes buscan trabajo con las vacantes disponibles y con las posibilidades de capacitación.

América Latina y el Caribe tienen alguna experiencia, aunque de alcance limitado, en la reforma y reestructuración de los sistemas de intermediación laboral. En este artículo se intenta relacionar esa experiencia con la de los países de la Organización de Cooperación y Desarrollo Económicos (OCDE), para determinar en qué medida los sistemas de esta índole podrían hacer un aporte relevante a las economías latinoamericanas y caribeñas. En primer lugar se examinará el concepto moderno de intermediación laboral y, en líneas generales, los numerosos servicios que abarca. A continuación se analizarán los procesos de reforma y modernización de los sistemas de intermediación en América Latina y el Caribe, haciendo hincapié en las recomendaciones y adaptaciones de mayor interés para las economías de esta región.

\section{II}

\section{Hacia un mayor entendimiento de la intermediación laboral}

Los servicios de intermediación laboral apuntan a aumentar la rapidez y la calidad del proceso de cuadrar los interesados en conseguir trabajo con las vacantes y la capacitación. Por consiguiente, estos servicios "median" entre la oferta y la demanda de trabajo. Sus principales usuarios son los desempleados, los subempleados y las empresas en busca de personal. 
La coordinación más rápida, más barata y de mejor calidad entre las personas que buscan empleo y los puestos vacantes tiene numerosas ventajas. En este caso, por mejor calidad se entiende que el empleado sea idóneo para el puesto, sea más productivo y, por ende, tenga mayores probabilidades de permanecer en él por más tiempo. La mayor rapidez y el menor costo se traducen en los siguientes beneficios: para la empresa, la contratación más rápida de una persona para llenar una vacante permite, entre otras cosas, reducir las pérdidas de producción, aumentar la productividad y disminuir las horas de trabajo dedicadas a la administración de personal; para el trabajador, la rápida obtención de un empleo se traduce en un mayor nivel de ingresos y un menor costo familiar y social por concepto de desempleo y subempleo. Este menor costo también beneficia a la comunidad en general, ya que reduce la necesidad de servicios sociales y las solicitudes de seguro de desempleo y seguridad social. En términos esquemáticos, los principales beneficios de los servicios de intermediación laboral son los siguientes:

- Reducción del desempleo de corto plazo y de la rotación laboral.

- Incremento de la productividad.

- Flexibilización de la movilidad laboral.

- Mejoramiento de la seguridad social.

- Mayor transparencia del mercado laboral y de la información y menor discriminación.

Para brindar los beneficios enumerados en los servicios de intermediación laboral se ha establecido un conjunto de servicios "básicos", presentes en todos estos sistemas, y que se describen a continuación:

i) Asistencia en la búsqueda de empleo. Prepara a la persona que busca empleo para encontrar un nuevo puesto. Puede incluir la redacción de currículos, elaboración de una estrategia para buscar empleo, información sobre el mercado de trabajo y participación en un "club de empleo". Los estudios realizados en los países de la OCDE siguen indicando que, en función de los costos, la asistencia para buscar empleo es unas tres veces más eficaz que la capacitación para ayudar a insertarse en el mercado laboral. También han demostrado tener efectos positivos los servicios de orientación, sobre todo si se les dedican dos o más sesiones.

ii) Servicio de colocación. Cuenta con un registro de puestos vacantes y lleva a cabo las actividades necesarias para encontrar, entre los interesados en conseguir trabajo inscritos en el servicio, las personas adecuadas para esos puestos. No es una tarea fácil de hacer bien. Las necesidades de los empleadores cambian rápida- mente y el registro deberá, para funcionar de manera adecuada, contar con un número amplio de listados, mantenerlos siempre actualizados y demostrar competencia al colocar a gente idónea en los puestos pertinentes, para que los empleadores sigan recurriendo al servicio.

Con el transcurso del tiempo, a partir de las funciones básicas de búsqueda de empleo y colocación, los servicios de intermediación laboral han desarrollado una gama de servicios secundarios diseñados para aumentar la calidad y la eficiencia de la intermediación. Estos servicios se definen como secundarios sólo porque no todos los sistemas de intermediación laboral los ofrecen, pero pueden resultar fundamentales para que la coordinación entre trabajadores y puestos funcione de manera eficaz. Los servicios secundarios principales son los siguientes: perfiles del empleo y evaluación de las competencias del postulante; capacitación directa o envío a capacitación; información sobre el mercado de trabajo; provisión de servicios sociales y de seguro de desempleo, y prestación de servicios especializados a los empleadores (cuadro 1) Algunos servicios de intermediación también se ocupan de las normativas de salud y seguridad en el lugar de trabajo, tema controvertido que puede generar enfrentamientos con los empleadores y dificultar los vínculos indispensables para una intermediación eficaz.

El examen de los acomodos recíprocos entre la base de usuarios, los servicios y su costo en un país y un contexto institucional dados es otra vía para apreciar mejor la gran variedad de sistemas de intermediación laboral existentes. Ningún conjunto de servicios puede satisfacer las necesidades de todos los que recurren a la intermediación laboral para encontrar trabajo. Algunos sólo necesitan asistencia mínima, otros necesitan mucho más. Puesto que, como en todo sistema, los recursos son limitados, se obtendrán mejores resultados concentrando los esfuerzos en quienes necesiten más servicios.

En el cuadro 2 se muestra una clasificación en tres categorías de las necesidades de los usuarios. Cuanto más alta la categoría, más servicios personalizados se requerirán para que el trabajador consiga empleo. A modo de ejemplo, la primera categoría, la más simple, es la de los usuarios "listos para conseguir trabajo" (categoría 1), que cuentan con calificaciones y antecedentes laborales recientes como para obtener un nuevo puesto con bastante facilidad. Puede tratarse de profesionales en actividades muy solicitadas o de comerciantes calificados. No se deberán desestimar los servicios destinados a esta categoría, ya que las investigaciones 
CUADRO 1

\section{Servicios de intermediación laboral: funciones principales}

\begin{tabular}{|c|c|c|}
\hline Categoría de servicio & Clientes destinatarios & Tipos de servicios \\
\hline $\begin{array}{l}\text { Búsqueda de empleo/perfil del } \\
\text { empleo }\end{array}$ & Los que buscan trabajo & $\begin{array}{l}\text { Evaluación de competencias o envío a evaluación } \\
\text { Caracterización de los usuarios para determinar los servicios que } \\
\text { necesitan } \\
\text { Elaboración de currículos } \\
\text { Orientación laboral } \\
\text { Bancos telefónicos para la búsqueda de empleo } \\
\text { Asistencia en la búsqueda de empleo } \\
\text { "Clubes de empleo" } \\
\text { Gestión de los casos individuales }\end{array}$ \\
\hline Colocación /intermediación & $\begin{array}{l}\text { Empleadores } \\
\text { Los que buscan trabajo }\end{array}$ & $\begin{array}{l}\text { Base de datos de vacantes a nivel nacional } \\
\text { Colocación } \\
\text { Revisión de listas de vacantes (empresas) } \\
\text { Selección de candidatos (para las empresas) } \\
\text { Recontratación por otra empresa (outplacement) } \\
\text { Selección de candidatos para puestos determinados (empresas) }\end{array}$ \\
\hline Capacitación & $\begin{array}{l}\text { Los que buscan trabajo } \\
\text { Proveedores de capacitación } \\
\text { Empleadores }\end{array}$ & $\begin{array}{l}\text { Evaluación de necesidades y requisitos de capacitación } \\
\text { Remisión a proveedores de capacitación públicos y privados } \\
\text { Capacitación directa por el sistema de intermediación laboral } \\
\text { (limitada) }\end{array}$ \\
\hline $\begin{array}{l}\text { Servicios especializados para } \\
\text { los empleadores }\end{array}$ & Empleadores & $\begin{array}{l}\text { Evaluación de recursos humanos } \\
\text { Asesoría legal en materia de empleo } \\
\text { Selección y examen de candidatos } \\
\text { Vinculación/enlace con promotores del sector privado } \\
\text { Orientación de la capacitación del personal }\end{array}$ \\
\hline $\begin{array}{l}\text { Información sobre el mercado } \\
\text { laboral }\end{array}$ & $\begin{array}{l}\text { Gobierno (local y nacional) } \\
\text { Empleadores } \\
\text { Los que buscan trabajo } \\
\text { Instituciones de capacitación }\end{array}$ & $\begin{array}{l}\text { Provisión de información y análisis de las tendencias del } \\
\text { mercado laboral }\end{array}$ \\
\hline $\begin{array}{l}\text { Seguro de desempleo/acceso a } \\
\text { los servicios sociales }\end{array}$ & $\begin{array}{l}\text { Los que buscan trabajo/ } \\
\text { trabajadores }\end{array}$ & $\begin{array}{l}\text { Administración de los beneficios del seguro de desempleo o } \\
\text { remisión al seguro de desempleo } \\
\text { Remisión a, o coordinación con, los servicios sociales } \\
\text { Remisión a programas de trabajo por cuenta propia }\end{array}$ \\
\hline
\end{tabular}

CUADRO 2

Usuarios (trabajadores) y servicios requeridos

\begin{tabular}{lll}
\hline$N^{\circ}$ & Descripción del usuario & Principales servicios requeridos \\
\hline 1 & Listo para conseguir trabajo & Únicamente servicios de orientación y colocación \\
2 & $\begin{array}{l}\text { En riesgo de estar desempleado o subempleado por un } \\
\text { plazo mediano }\end{array}$ & $\begin{array}{l}\text { Evaluación de competencias y orientación laboral previas a la } \\
\text { colocación o la inscripción en un cursillo de capacitación }\end{array}$ \\
3 & $\begin{array}{l}\text { En alto riesgo de estar desempleado o subempleado } \\
\text { por un plazo largo }\end{array}$ & $\begin{array}{l}\text { Desarrollo de un plan de empleo y capacitación de largo plazo, } \\
\text { seguimiento más sistemático, intervención y servicios sociales }\end{array}$
\end{tabular}

siguen señalando que la asistencia para buscar trabajo es uno de los servicios más eficaces que prestan los sistemas de intermediación laboral, sobre todo en función de los costos por usuario. Igual que en los programas relativos a mercados de trabajo en general, lo que es esencial es no dar estos servicios a quienes pueden encontrar trabajo con igual facilidad y rapidez sin asistencia (es decir, hay que evitar la pérdida por "peso muerto").

Por último, es fundamental no confundir los servicios de intermediación laboral con las firmas, de naturaleza muy diferente, que actúan como empleadores intermediarios y son denominados "servicios" o "intermediarios" en algunos países latinoamericanos. 
En países como Perú y Bolivia, las empresas contratan a firmas "de servicios" para que empleen los trabajadores que necesitan, evadiendo así las normas laborales y beneficios que se aplican a los contratados con carácter permanente. Esta modalidad tiene graves con- secuencias para la protección legal de los trabajadores. La intermediación laboral abarca los servicios destinados a la contratación directa de los trabajadores en las empresas, no la contratación indirecta a través de intermediarios.

\section{III}

\section{Países de la OCDE: tendencias y reformas recientes}

Durante más de una década, los países de la ocDE han reexaminando y reformado conjuntamente el papel de los servicios de empleo públicos y privados en el marco de una combinación de políticas "pasivas" y "activas" vinculadas a los mercados nacionales de trabajo. Estos esfuerzos no están únicamente orientados a la reforma de los servicios públicos de empleo existentes, sino también a la modernización y al diseño de un "sistema" más amplio de servicios públicos y privados capaces de responder a las exigencias de un mercado global. Estos servicios renovados de intermediación laboral están aprovechando las nuevas tecnologías y exigiendo resultados más explícitos. En esta sección se examinan cuatro principales tendencias y reformas.

\section{Integración y vinculación de las políticas sobre mercados laborales}

En muchos países de la OCDE se comprobó la ausencia de un vínculo eficaz y sinérgico entre las principales funciones de política del mercado laboral: el empleo, la capacitación y la intermediación. A menudo estas funciones competen a diferentes organismos, o se llevan a cabo aisladamente, pese a que las tres se hallan extremadamente relacionadas. Es preciso contar con información sobre los puestos vacantes y las necesidades del sector privado para garantizar que los programas de capacitación y de empleo por cuenta propia se adecuen a las demandas reales. Una estrecha coordinación entre la colocación y la capacitación asegura que los desempleados y subempleados adquieran las habilidades necesarias para ocupar las vacantes efectivamente disponibles. El seguro de desempleo y otras medidas de apoyo al ingreso deben acompañarse de una búsqueda real de trabajo, para que cumplan con la función para la cual fueron concebidos: amortiguar la pérdida de ingresos en el período de búsqueda de un nuevo puesto. Esto disminuye los casos en que el seguro de desempleo disuade al trabajador de la búsqueda de un nuevo empleo, o la retrasa.

Algunas de las principales vías que han empleado los países de la OCDE para estrechar el vínculo entre empleo/seguro de desempleo, capacitación e intermediación son las siguientes:

- Agregar en los servicios nacionales de intermediación la función de enviar a capacitación.

- Unificar las prestaciones de los seguros de desempleo y los servicios de colocación o, por lo menos, coordinarlos más eficazmente.

- Crear un servicio integrado que unifique y simplifique todas las funciones básicas.

No es esencial tener el seguro de desempleo y la intermediación laboral bajo un mismo techo, ya que cada país tiene tradiciones e inquietudes propias que determinan si la unificación del seguro de desempleo y la intermediación laboral disuade o no de recurrir al servicio de colocación a quienes no reciben los beneficios del seguro, y si la prestación del seguro de desempleo otorga o no al servicio de intermediación un enfoque más propio de un servicio social. En Japón, por ejemplo, se intenta fortalecer la vinculación mediante la rotación del personal entre las prestaciones del seguro y el servicio de empleo. La clave es lograr una coordinación eficaz en el contexto particular de cada país.

La ventanilla única (one-stop shop) ha representado una innovación en ciertos países, entre otros los Estados Unidos, donde permitió ampliar el acceso en línea a toda una gama de servicios de empleo. Los programas de compensación de ingresos, entre los que se cuenta el seguro de desempleo, tienen menor difusión en América Latina y el Caribe, pero para la región resulta interesante la incorporación en los centros de intermediación de servicios directos de empleo por 
cuenta propia o de envío a servicios de esa índole, así como el suministro de más recursos.

\section{Introducción de las señales del mercado y mayor papel de las agencias de empleo pri- vadas}

Tras el fin del monopolio público de los servicios de empleo, la mayoría de los países de la OCDE ha ido incorporando una gama de mecanismos de mercado para hacer que el desempeño de los servicios públicos sea más parecido al de las empresas privadas. Estas reformas se pueden catalogar en dos grupos: i) las que apuntan a elevar la eficacia de los servicios públicos de empleo mediante mecanismos de mercado y ii) las que buscan desarrollar la competencia y la asociación con agencias de empleo privadas. El principio que subyace a estas reformas basadas en el sector privado es la coincidencia en la necesidad de separar los papeles del gobierno como proveedor y como contratante de servicios. Por ende, en la mayor parte de los servicios de intermediación se está dejando de proporcionar capacitación para permitir que el usuario elija proveedores de capacitación que compitan entre sí.

Se ha inducido a los servicios públicos a actuar de manera más similar a las empresas privadas, recurriendo, por ejemplo, a la contratación externa y la licitación competitiva de la capacitación activa para el mercado de trabajo, que resulta esencial; estableciendo tarifas para los usuarios y servicios pagados, y usando sistemas de vales. En Suecia, el servicio público de empleo contrata cursos con otros centros de capacitación del sector público y del sector privado. En el Reino Unido, los consejos de capacitación y empresa locales que administran los servicios de empleo contratan a agentes externos para evaluar las necesidades de capacitación, los que a su vez contratan gestores para brindar la capacitación, o para contratarla con otros proveedores. Los vales constituyen otro método para estimular la competencia. Los beneficiarios reciben un vale para pagar la capacitación que efectúan proveedores externos, de manera que es esencial que reciban mucha información sobre el mercado de trabajo y los cursos de capacitación existentes y que se supervise la puesta en práctica del sistema. En los servicios de empleo, tanto públicos como privados, el cobro al usuario se ha visto limitado en gran parte debido a las restricciones impuestas por el Convenio $\mathrm{N}^{\circ} 96^{1}$ de la Organi-

${ }^{1}$ Convenio sobre las agencias retribuidas de colocación (revisado) (1994). zación Internacional del Trabajo (OIT), que prohibió el cobro de honorarios a los trabajadores. Se ha interpretado que estas restricciones no se aplican a las tarifas que los servicios públicos de empleo cobran a los empleadores por algunos de los nuevos servicios especializados, por lo que este cobro se ha hecho cada vez más común. En Bélgica, los empleadores pagan por el servicio adicional de selección previa exhaustiva de los candidatos y por los servicios de reinserción laboral. En los Países Bajos, la institución de una serie de servicios retribuidos para los empleadores puso a las oficinas de intermediación locales en un contacto más directo con los empleadores, lo que se tradujo en beneficios más amplios tanto para el servicio como para las personas en busca de trabajo.

Un segundo conjunto de reformas basadas en el mercado apunta a abrir el mercado a las agencias de empleo privadas y a las organizaciones comunitarias de colocación y servicios de empleo. Esta expansión abarca esfuerzos dirigidos a:

- Flexibilizar las restricciones legales a los servicios privados de empleo, para permitir la competencia con los servicios públicos;

- Contratar directamente con agencias de empleo privadas y organizaciones comunitarias la colocación de personas (como en Australia) y estimular la competencia.

- Establecer acuerdos de asociación con agencias y organizaciones privadas, para lograr una mejor cobertura del mercado de colocaciones.

Contrariamente a lo esperado, la apertura del mercado de los servicios de empleo en numerosos países no condujo a un aumento notorio de la participación del sector privado. Esto se debe probablemente a que este sector atiende un mercado más reducido y de alto nivel y a que se necesita un considerable período inicial y de consolidación de prestigio para lograr una participación importante (así sucedió en Dinamarca a principios del decenio de 1990).

\section{Incorporación y fortalecimiento de los indicadores de desempeño y de las reformas de la gestión}

Los países de la OCDE han emprendido ampliamente la tarea de mejorar el desempeño interno de los servicios de intermediación laboral sobre la base de nuevas medidas de rendimiento y reformas de la gestión. Para medir el cumplimiento de las metas de esos servicios se ha ido avanzando desde el uso de indicadores retrospectivos de desempeño al uso de indicadores pros- 
pectivos del funcionamiento del servicio, y se incluyen metas en cuanto a "vacantes cubiertas", "vacantes cubiertas con participación activa del servicio" y "tiempo requerido para cubrir una vacante". Con el fin de mejorar el desempeño se pueden utilizar premios y castigos, como por ejemplo: medidas disciplinarias, inspecciones de gestión, primas presupuestarias y otros incentivos y sanciones. En Suiza, el gobierno nacional clasifica las oficinas de colocación por su desempeño teniendo en cuenta influencias externas como las condiciones del mercado laboral, y paga a cada cantón o gobierno regional el monto correspondiente al desempeño de la oficina de colocación que administra.

Los indicadores de desempeño interno tienen claras limitaciones. En primer lugar, de no emplearse indicadores más sofisticados, no se medirán los efectos de un servicio o curso de capacitación determinado, sino solamente su volumen y si fue puesto en práctica de manera eficiente. Es posible que un servicio local parezca estar cumpliendo con los objetivos de colocación, pero quizás se trate de empleos transitorios o inadecuados para los trabajadores seleccionados. Otro servicio tal vez dedique más tiempo a cada usuario, con lo cual su tasa de colocación puede ser menor pero quizás tenga una mayor incidencia en el desempleo local. En el Reino Unido, un comité de la Cámara de los Comunes ha recomendado que se remplacen las metas basadas en la colocación por otras que representen el "valor agregado" a través de la satisfacción del usuario, teniendo en cuenta que los sistemas de intermediación laboral están brindando cada vez más servicios adicionales a los de colocación. Los indicadores de desempeño interno deberían complementarse o integrarse dentro de un sistema de evaluación más amplio que analice los efectos de mayor magnitud o de más largo plazo. En suma, no se puede depender solamente de los indicadores del desempeño interno para aumentar la eficiencia y la eficacia. Por lo tanto, el desarrollo de indicadores de desempeño generalmente ha formado parte de reformas de gestión y cambios internos más ambiciosos, concebidos para incrementar la eficacia y la cobertura globales de los servicios de intermediación.

Las principales reformas de gestión adoptadas por los países de la OCDE incluyen: la descentralización, la gestión por objetivos y un mayor uso de las tecnologías de la información. La descentralización de los recursos y la flexibilidad de los programas se han empleado para mejorar la capacidad de adaptar los servicios a las necesidades de la economía local (por ejemplo en Francia). La gestión por objetivos reforma toda la estructura de incentivos al personal y de desempeño. En Austria, Finlandia y Suecia se ha estado experimentando con este tipo de cambios. En estos casos, se ha otorgado a las oficinas locales mayores facultades para distribuir sus presupuestos entre los diversos servicios (entre otros, búsqueda de trabajo, capacitación y subsidios de empleo) y para ajustarse a los objetivos acordados. Por último, las tecnologías de la información representan probablemente el cambio en materia de gestión que se ha impuesto más ampliamente en los países de la OCDE, con el fin de aumentar la eficacia y la cobertura de los servicios de empleo. En numerosos países los "bancos de trabajo" se han puesto en línea, incorporando al sistema una gama de usuarios mucho mayor, que prefiere emplear sistemas de autoservicio desde sus casas, centros comunales o incluso centros comerciales.

\section{La focalización en los desempleados de largo plazo}

Por último, un conjunto de reformas en el que han hecho hincapié casi exclusivamente los países industrializados de altos ingresos es el de aquellas encaminadas a lograr que los servicios de intermediación laboral respondan mejor a las necesidades de los casos más difíciles, es decir, de quienes han estado desempleados de manera continua durante doce meses o más. Estas reformas propician intervenciones de política más tempranas en el caso de los usuarios con mayor riesgo de estar desocupados y la focalización de mayor cantidad y variedad de servicios en los desempleados de largo plazo. Ellas son las siguientes:

- Diagnóstico e intervención temprana. Identificar tempranamente a los usuarios en mayor riesgo a través de entrevistas y programas computacionales, y focalizar en ellos los servicios antes de que el desempleo de largo plazo sea un hecho.

- Gestión de casos. Abordar en forma individualizada una estrategia de reinserción laboral para cada caso y adecuar los servicios a las necesidades particulares del usuario.

- Exigencias para percibir el seguro de desempleo y secuencia de las prestaciones. Hacer más estricta la exigencia de que efectivamente se busque trabajo de manera activa como condición para percibir el seguro de desempleo, y establecer una secuencia de servicios encaminados a lograr el retorno del desempleado al mercado de trabajo de la forma más rápida y duradera posible. 


\section{IV}

\section{Tendencias de los sistemas de intermediación laboral en América Latina y el Caribe}

Como en la mayor parte del mundo en desarrollo, las reformas y la modernización de los sistemas de intermediación laboral en América Latina y el Caribe han sido más limitadas que en la OCDE. A muy grandes rasgos, en esta región predomina un modelo de intermediación del sector público que, debido a las restricciones de recursos, es habitualmente reducido y atiende al estrato inferior de la fuerza de trabajo. En Centroamérica, por ejemplo, los directores de los servicios públicos de empleo sostienen que allí los servicios de intermediación se han dedicado al estrato de baja calificación técnica, en los niveles inferiores de la pirámide ocupacional (OIT, 1998, p. 2). Por regla general, los servicios nacionales de empleo de la región se administran a través de los ministerios de trabajo o institutos de capacitación de la órbita estatal, como el Servicio Nacional de Aprendizaje (SENA) en Colombia.

La motivación para la reforma y el cambio en esos sistemas tiene rasgos propios en América Latina y el Caribe. En la OCDE las reformas fueron impulsadas por un mal desempeño y por la necesidad de reducir costos y, en especial, de llegar a los casos peores de desempleo persistente. En América Latina y el Caribe las necesidades económicas son diferentes, pero los sistemas de intermediación también han sido criticados de manera similar, reprochándoseles malos resultados y, sobre todo, politización. En esta región, particularmente, mientras que en teoría el monopolio del sector público apuntaba a proteger y asistir a los trabajadores más necesitados de ayuda, en la práctica las inversiones del sector público nunca fueron lo suficientemente cuantiosas como para cubrir una porción importante del mercado de trabajadores. Muchos servicios públicos de empleo latinoamericanos y caribeños adquirieron la reputación de tener en sus registros predominantemente puestos de menor calificación y un bajo número de trabajadores, de modo que los grupos menos favorecidos rara vez pudieron tener información sobre empleos de mayor calificación y mejor pagados, o acceder a esos empleos, a través de esos servicios públicos.

Puesto que en la región latinoamericana y del Caribe la inversión pública en política laboral es baja y el seguro de desempleo excepcional, la reducción del costo no reviste tanta importancia. Los países de esta región podrían emprender la reforma de los sistemas de intermediación laboral con el fin de lograr más transparencia en mercados de trabajo ineficaces $y$, sobre todo, de aminorar la discriminación en el mercado laboral. La búsqueda de trabajo en la región sigue dependiendo en gran medida de redes informales y de contactos familiares y personales que pueden conducir a claras fallas de productividad y a fortalecer y perpetuar la discriminación vinculada a la raza, el origen étnico, el género y el nivel económico.

En la región, los sistemas de intermediación laboral exhiben mucho menor desarrollo que los de la OCDE y enfrentan exigencias muy diferentes del mercado laboral. Algunas de las principales diferencias que afectan la forma en que deberían reestructurarse los sistemas de intermediación son las siguientes:

- Los sistemas de intermediación en América Latina y el Caribe son de tamaño reducido y su experiencia es limitada; muchos sufren las consecuencias de años de escasa inversión pública.

- Las empresas del sector privado que prestan servicios de colocación y empleo son pocas, de aparición reciente y normalmente se dedican en forma exclusiva a los empleos de nivel más alto.

- El empleo en el sector informal puede superar el $50 \%$ de la economía nacional, y concentra a los trabajadores pobres y menos favorecidos. Esto determina, inevitablemente, un enfoque diferente de la intermediación laboral, para reflejar una realidad caracterizada por el predominio del empleo por cuenta propia, sobre todo en las áreas rurales.

- Para los sistemas de intermediación de América Latina y el Caribe, los casos más difíciles no son los de desempleo prolongado, como en la OCDE, sino los de subempleo grave y baja calificación.

- La mayoría de los países latinoamericanos carece de seguro de desempleo u otra forma de compensación de ingresos para los desempleados, de modo que las reformas de los sistemas de intermediación laboral dejan de lado el problema de la asistencia a los trabajadores desempleados. 
En los países de la región en los que sí existe seguro de desempleo, entre otros Argentina y Brasil, éste generalmente no se administra a través del servicio de empleo. Por ende, la posibilidad de reducir el costo del seguro no es un incentivo para reformar los servicios nacionales de empleo en América Latina y el Caribe, como sí lo es en muchos países de la OCDE.

- La considerable migración interna y externa que exhiben varios países (por ejemplo, algunos países centroamericanos y México, entre otros) crea necesidades adicionales de intermediación.

Con las diferencias anotadas, las reformas y revisiones de la intermediación laboral realizadas hasta la fecha en América Latina y el Caribe se han centrado principalmente en: i) la adopción de sistemas informáticos, ii) la ampliación del papel del sector privado y iii) el mejoramiento del desempeño y la gestión.

\section{Sistemas de información y sistemas basados en Internet: adopción y expansión}

En toda la región se percibe una tendencia clara a utilizar las nuevas tecnologías de información para mejorar el desempeño de los sistemas de intermediación laboral. La inversión en tecnología informática para la intermediación ha sido más restringida en América Latina y el Caribe que en la OCDE, pero es apreciable en países como Brasil, México, Chile y Costa Rica. La modernización de los sistemas de información incluye: bolsa de trabajo y servicio de colocación, ambos en línea, sistemas internos de información en las oficinas, y sistemas de información sobre el mercado laboral. El Servicio Nacional de Capacitación y Empleo (SENCE), de Chile, ha establecido la primera bolsa electrónica de trabajo del país. En InfoEmpleo hay más de veintinueve mil currículos de personas que buscan empleo, y se reciben más de trescientas notificaciones de vacantes al mes. En Costa Rica, gracias a la financiación y el apoyo del Instituto Nacional de Aprendizaje (INA), se está desarrollando un sistema de información en dos etapas para revitalizar el servicio público de empleo, ampliarlo a una red de proveedores privados y sin fines de lucro y crear un registro nacional de empleo cuyo servidor central será responsabilidad del INA. En la región es importante también contar con bolsas de trabajo electrónicas e información relativa a los mercados de trabajo a nivel regional. En virtud de un proyecto del Fondo Multilateral de Inver- siones del Banco Interamericano de Desarrollo (BID), ocho países centroamericanos, incluidos la República Dominicana y Panamá, están desarrollando un sistema de información sobre el mercado laboral para compartir y analizar con criterio regional datos sobre el mercado de trabajo que abarquen información sobre las tendencias del empleo, la economía y la ocupación.

\section{Papel del sector privado y asociación con ins- tituciones privadas y sin fines de lucro}

En la región se observa una importante tendencia a la expansión de los servicios de intermediación laboral privados y sin fines de lucro y de los vínculos entre ellos y los proveedores públicos. La primera etapa de este proceso es la apertura al sector privado y su legitimación y reglamentación. Desde el decenio de 1980, varios países de la región están trabajando en la constitución de marcos legales apropiados para los proveedores privados y sin fines de lucro y en el establecimiento de normas para su supervisión. En Panamá, por ejemplo, se aprobó una ley (agosto de 1995) que autorizó las agencias de empleo con fines de lucro y asignó su supervisión al Ministerio de Trabajo.

En la segunda etapa, más importante, se fomentará la expansión de las agencias de empleo privadas y la creación de nuevas asociaciones entre proveedores públicos, privados y sin fines de lucro. En 1996, en Perú se dio un renovado impulso al servicio de empleo público mediante la creación de una red de centros privados y sin fines de lucro, estimulando el ingreso de una gran cantidad de proveedores nuevos e innovadores al negocio de la intermediación laboral. Estos Centros de Intermediación Laboral (CIL) incluyen centros de capacitación, centros comunales locales y organizaciones afiliadas a agrupaciones religiosas, cada uno con sus contactos y conocimiento a nivel local de los empleadores y de los que buscan trabajo. Aunque se deberán realizar evaluaciones adicionales, de un análisis primario del sistema peruano - la Red CILProEmpleo- se desprende que los proveedores públicos y privados que se han integrado a la red han logrado aumentar su cobertura y eficiencia. En Guatemala, con el apoyo del Banco Interamericano de Desarrollo, se están iniciando planes para crear una red de servicios de empleo (RESE), en la que las oficinas públicas locales del servicio nacional de empleo de cada región o provincia actuarán como centro de una red local que integrará a proveedores privados y sin fines de lucro. 


\section{Mejoramiento del desempeño y ampliación de la base de usuarios}

En los sistemas de América Latina y el Caribe se están adoptando medidas adicionales para mejorar el desempeño de los servicios de empleo, su producción y su base de usuarios, entre otras las siguientes:

Ferias de empleo como "ventanillas únicas" temporales. Las ferias de empleo se están empleando en la región con cometidos que trascienden el encuentro cara a cara por un día de empleadores y de personas en búsqueda de empleo. En estas ferias (las que se celebran en Panamá, por ejemplo) se ofrece también una gama de servicios a los que se accede de una sola vez mediante ventanillas únicas, lo que se asemeja a los one-stop shops de los países de la OCDE. Además de acceso a entrevistas con empleadores locales, los servicios disponibles en las ferias de trabajo panameñas suministran asistencia técnica a microempresas, orientación profesional, talleres de redacción de currículos, información sobre programas de capacitación y evaluación de competencias. También México, desde hace muchos años, tiene un enfoque global de las ferias de empleo.

Descentralización. En la región latinoamericana y del Caribe es más débil que en la OCDE la tendencia a descentralizar los servicios de intermediación laboral y ponerlos bajo la responsabilidad de las municipalidades y oficinas locales. El papel y la factibilidad de la descentralización en un país dado dependerán tanto de la existencia a nivel nacional de una estrategia y una tendencia más amplias orientadas a la descentralización, como de las capacidades de las instituciones locales. En Chile, de conformidad con la política nacional, la descentralización se ha llevado a cabo transformando las oficinas locales de empleo en una red de más de ciento cincuenta agencias de empleo municipales. Estas agencias brindan a los trabajadores servicios de intermediación laboral gratuitos. El Servicio Nacional de Capacitación y Empleo (SENCE) presta asistencia técnica a la red municipal, y planifica y supervisa los programas e instituciones de capacitación, pero las funciones centrales de intermediación corresponden al nivel local.

Perfeccionamiento de los servicios e instrumentos de evaluación. Numerosos países de América Latina y el Caribe están ampliando asimismo la gama de servicios específicos e instrumentos que ofrecen los centros de intermediación (por ejemplo, mejores metodologías de búsqueda de empleo, evaluación de competencias), y aspiran a que la información y los instrumentos sean compartidos por toda la región.

\section{$\mathrm{V}$}

\section{Modelos institucionales de servicios de intermediación laboral}

Las reformas y medidas de fortalecimiento en curso, tanto en la OCDE como en América Latina y el Caribe, deben considerarse dentro del contexto más amplio de la reforma de los propios marcos institucionales para la prestación de servicios de intermediación laboral. Es el cambio en los vínculos institucionales entre el gobierno, el sector privado y los actores sociales lo que determina las modificaciones más importantes de los servicios de intermediación laboral e incentiva con más fuerza a elevar el número de usuarios y de proveedores de información. La variedad de sistemas de intermediación laboral adaptados a circunstancias nacionales muy dispares es impresionante. Este estudio, en el que se pasó revista a tales sistemas a través del mundo, iden- tificó seis modelos. Estos modelos pueden entenderse, en términos sencillos, como un espectro lineal de posibilidades que van desde un servicio de empleo únicamente público en un extremo (con exclusión de todo servicio privado) hasta un sistema únicamente privado en el otro, con la salvedad de que pocos sistemas nacionales se hallan en uno u otro de estos dos extremos. Dentro de cada modelo hay variaciones de importancia entre un país y otro, sobre todo en la porción media del espectro, es decir, en las asociaciones entre los sectores público, privado y sin fines de lucro. Los dos primeros modelos, A y B, representan sistemas muy anteriores a las reformas y renovaciones. Los modelos más nuevos van de la $\mathrm{C}$ a la $\mathrm{F}$. 


\section{Modelo A: monopolio público}

Este modelo predominó en el período posterior a la segunda guerra mundial. El sector público tenía el monopolio legal para administrar un servicio de intermediación nacional, con el fin de garantizar que no se explotara a los trabajadores mediante el cobro de tarifas altas y que los trabajadores menos favorecidos tuvieran acceso a los servicios. Los convenios de la OIT sancionados en la década de 1940 prohibían la existencia de empresas privadas que cobraran por la colocación. Se pensaba entonces, y en muchos casos se sigue pensando hoy, que el papel del sector público en este campo era indispensable porque el libre mercado de servicios de empleo probablemente acarrearía la exclusión de los trabajadores más difíciles de colocar, con ingresos bajos y menos favorecidos, mediante un proceso de filtrado (creaming), por el cual el sector privado retendría únicamente a los trabajadores de más fácil colocación. La necesidad de que el sector público asuma ese papel tiene como fundamento teórico las imperfecciones del mercado que llevan a una insuficiente provisión de servicios de empleo para ciertos tipos de trabajadores.

En el marco del modelo basado en el sector público existe un único servicio público nacional, generalmente dependiente de un departamento de trabajo u otro órgano ejecutivo, al que todos los trabajadores pueden acceder de forma gratuita. Sólo se permiten los servicios de empleo privados gratuitos, las agencias de empleo temporal y las que atienden a trabajadores teatrales y artistas. En la práctica, el monopolio público del servicio de empleo fue eliminado formalmente en la mayoría de los países tras el convenio de la oit de 1997, y en convenios nacionales como en Portugal (1989) y en Finlandia y Austria (1994). ${ }^{2}$

Actualmente son pocos los países en los que la prohibición legal todavía está vigente. Y aun allí donde lo está, suelen surgir de todas maneras algunas agencias de empleo privadas. En Costa Rica las agencias de empleo privadas no están permitidas por ley, pero en la práctica funcionan en el país por lo menos 42 servicios de ese tipo, bajo el rótulo de empresas consultoras. Hoy —en los casos en que existe- este mo-

\footnotetext{
${ }^{2}$ Los convenios básicos pertinentes de la oIT son el Convenio sobre el servicio de empleo, de 1948, ratificado por quince países de América Latina y el Caribe; el Convenio sobre las agencias retribuidas de colocación, de 1949 (Convenio $\mathrm{N}^{\circ}$ 96), ratificado por 17 países de América Latina y el Caribe, y el Convenio sobre las agencias de empleo privadas, de 1997 ( $\left.\mathrm{N}^{\circ} 181\right)$, ratificado por un solo país de América Latina y el Caribe.
}

delo se puede describir como un monopolio del servicio público de empleo, que domina la prestación de servicios de intermediación, pero que tiene poca cobertura a nivel nacional, y que coexiste con agencias privadas que actúan a niveles limitados en un marco legal precario e incierto.

\section{Modelo B: Servicio nacional de empleo que compite de hecho con proveedores privados}

Este modelo es común en los países en desarrollo. Se permiten las agencias de empleo y los servicios de empleo privados, pero los servicios públicos y los privados actúan con casi total independencia recíproca. Los servicios de empleo privados pueden estar bajo la regulación del servicio público u otro organismo, o regirse únicamente por las leyes del mercado. La coexistencia de los proveedores públicos y privados obedece a la teoría de que, gracias a la introducción de una cierta dosis de competencia de los proveedores privados con los servicios públicos, mejorará la calidad de la intermediación y habrá más innovación en unos y otros. En la práctica, aunque efectivamente existe cierta competencia, generalmente no es muy intensa, ya que cada sector tiende a cubrir únicamente una parte del mercado nacional y sus usuarios casi no se superponen. La tendencia normal del mercado apunta a que el servicio público se dedique a los usuarios de menores ingresos y las empresas privadas a los profesionales y trabajadores de ingresos altos, más fáciles de colocar y con mayor capacidad de pago.

En Panamá, un reducido servicio nacional de empleo coexiste con unos pocos proveedores privados y no gubernamentales. La interacción entre los sectores público y privado es limitada. Esto empezará a cambiar cuando en la capital panameña se ponga en marcha una red formada por integrantes públicos y privados. A medida que ambos sectores establezcan vínculos más definidos, será posible poner en marcha formas más amplias de cooperación entre ellos, características de los modelos C y D.

\section{Modelo C: Asociaciones entre servicios de empleo públicos y privados}

La reforma y transformación de los servicios públicos de empleo a través del establecimiento de nuevos vínculos y asociaciones, no sólo con el sector privado sino también con actores sociales clave como sindicatos y organizaciones comunitarias, se han llevado a cabo por diversas vías. En este modelo, la asociación se define 
como una relación de colaboración, no competitiva, por la cual instituciones públicas y privadas comparten, entre otras cosas, recursos, información, servicios y usuarios. A medida que las necesidades de intermediación laboral se hacen más y más específicas, las asociaciones se vuelven un marco institucional cada vez más común, por razones esencialmente prácticas y no teóricas. Dado que el rol desempeñado en el mercado por los proveedores públicos y privados ha sido hasta la fecha relativamente modesto, se ha recurrido a las asociaciones como forma de aumentar a la vez la cobertura de mercado de los proveedores tanto públicos como privados, y obtener mayores economías de escala. Cabe notar que parece haber pruebas de que los proveedores públicos y privados pueden modificar positivamente la curva de demanda de la intermediación mediante una mayor colaboración y la puesta en común de sus listas, aunque la eficacia de cada proveedor para colocar a quienes buscan trabajo pueda ser muy diferente.

Se examinarán aquí tres tipos de asociación colaborativa. En el primer tipo, el servicio de empleo público está constituido por una red de proveedores públicos, privados y sin fines de lucro que da origen a un sistema nacional más amplio y eficaz. El caso de Perú ofrece un ejemplo interesante. En 1996, el Ministerio de Trabajo de ese país lanzó una red de proveedores sin fines de lucro y privados, que cubría principalmente las áreas urbanas. Representantes de las iglesias, las organizaciones sociales y los centros de capacitación pudieron postular a convertirse en oficinas locales de intermediación e información laboral. En todas las oficinas de la red se inscribe a los que buscan trabajo, se brinda asistencia para buscar empleo, se coloca a las personas en puestos vacantes locales y se accede a la base de datos nacional de vacantes. Incluso en los primeros años de aplicación se pudo apreciar un aumento claro, año tras año, de los inscritos tanto en los proveedores públicos como en los privados, de los puestos listados y, lo que es más importante, del porcentaje de colocación de trabajadores. De 1998 a 2000, las colocaciones sumadas de los centros públicos y privados se incrementaron en un $28 \%$, aumentó la eficiencia (medida por el porcentaje de las personas en busca de empleo que fueron colocadas) y subieron las vacantes cubiertas (Ministerio del Trabajo y Promoción Social, 2001). En este tipo de redes, el servicio público puede seguir siendo una entidad independiente, como en Perú, o ser reemplazado por una red de oficinas locales y regionales, como en el caso de Chile.
Un segundo tipo de asociación apunta a suministrar lineamientos de política y supervisión para mejorar la puesta en práctica de los servicios de intermediación laboral y la coordinación general de las políticas relativas al mercado de trabajo. México creó en 1992 comités ejecutivos de sus oficinas de empleo estaduales, encargados de orientar las operaciones de esas oficinas. Estos comités están integrados por representantes de asociaciones empresariales y empresas; de instituciones de capacitación; de organismos gubernamentales, estaduales y federales, y de sindicatos. A su vez, dichos comités participan en los consejos estaduales de productividad y competitividad, encargados de coordinar las políticas relativas al mercado laboral entre los gobiernos estaduales y el gobierno federal.

Un tercer tipo de asociación entre instituciones públicas y privadas se ha concebido para atender a los grupos de personas menos favorecidas o con necesidades especiales. En el Reino Unido existe un ejemplo interesante del uso de asociaciones a nivel local para servir mejor a los grupos a los que cuesta más llegar. Las responsabilidades y la flexibilidad para generar estas asociaciones adaptadas a las necesidades locales son de competencia local. Las del Reino Unido se formaron para prestar asistencia a grupos de personas discapacitadas, a grupos minoritarios y a personas con serias dificultades de aprendizaje.

\section{Modelo D: Servicios autónomos tripartitos y participación de actores sociales clave}

Una forma más sofisticada de asociación se generó con el reemplazo del servicio público de empleo por organizaciones autónomas constituidas por actores sociales clave. Estos servicios no son ni exclusivamente públicos, ni exclusivamente privados; funcionan con autonomía, y toman todas las decisiones fundamentales respecto de la actuación de las oficinas y la provisión de los servicios. Generalmente reciben una importante financiación pública, a través de subsidios y el pago de servicios de capacitación y otros, pero la administración es independiente del sector público. En un estudio reciente se concluye que casi en la mitad de los países de la OCDE existen actualmente servicios de empleo tripartitos autónomos. Estos pueden administrarse sobre una base tripartita integrada por representantes de los medios empresarial, laboral y gubernamental (local y federal), como en Alemania, o sobre la base de diferentes agrupaciones de actores sociales. En los Países Bajos se inició una organización tripartita en 1991, pero el gobierno decidió retirarse de su ad- 
ministración en 1996 y dejar en su lugar a profesionales independientes, ya que los representantes gubernamentales se habían encontrado en posiciones políticas difíciles y comprometedoras, que a menudo paralizaron la toma de decisiones.

La ventaja clave de este modelo radica en la participación más activa de las partes directamente interesadas a nivel local y en una mayor rapidez y flexibilidad para tomar decisiones. Se espera que los servicios autónomos tengan mayor cobertura de las oportunidades laborales y registros más amplios de postulantes a empleos. Para que estos servicios puedan funcionar de manera eficaz y sin contratiempos, tienen que haberse establecido previamente vínculos de cooperación entre los sectores sindical, gubernamental y privado, de modo de evitar la polarización y la ineficacia en la toma de decisiones. En el Reino Unido se intentó establecer un sistema tripartito, pero la administración Thatcher volvió a poner el servicio en el ámbito del sector público, tras graves disputas con los participantes sindicales. El consenso entre el gobierno y los actores sociales, y la existencia previa de vínculos fuertes, son elementos clave para el éxito de los servicios autónomos. Por lo tanto, el modelo es menos aplicable a las economías en desarrollo, en las que los vínculos de gestión están menos desarrollados.

\section{Modelo E: Red basada en la competencia, con financiación pública}

Entre los países de la OCDE, Australia está realizando reformas audaces para crear un modelo propio y diferente. El sistema australiano ha emanado de reformas anteriores a un enfoque de "gestión de casos", según el cual el servicio de empleo público contrataba proveedores privados de servicios de colocación y otros servicios. En 1998 se inició una reforma más profunda en materia de contratación externa, con miras a la "provisión competitiva de servicios". En este nuevo modelo el servicio público de empleo fue remplazado por una "red" de servicios de empleo competitiva integrada por 310 organizaciones privadas, comunitarias y gubernamentales, que reciben un pago individual por la colocación de sus usuarios. Las funciones de esta red, esbozadas en la página de Australia en Internet, abarcan la intermediación, el empleo y la capacitación. Este nuevo modelo institucional está fuertemente determinado por los logros, puesto que los organismos (ya sean públicos, privados, o sin fines de lucro) reciben un monto determinado por cada desempleado que colocan, y un monto extra si la persona había estado desempleada por largo tiempo. En la segunda licitación el servicio público de empleo fue reducido, ya que las organizaciones sin fines de lucro administradas por iglesias y otras organizaciones de voluntarios fueron las que más colocaciones concretaron. El modelo de red de servicios de empleo competitivo fue concebido para superar los defectos de los modelos anteriores, que desatendían las necesidades de los menos favorecidos. En el modelo australiano se entregan recursos adicionales a los proveedores públicos y privados como incentivo para atender a estos últimos. Sería precipitado pronunciarse en este momento sobre la eficacia del nuevo modelo; sin embargo, un legislador británico declaró que se trataba de "una iniciativa única en su género que está siendo seguida de cerca por los ministros de trabajo del mundo entero".

\section{Modelo F: Servicios basados en el sector privado}

En este modelo no existen los proveedores públicos. Sin embargo, el sector público puede proporcionar financiamiento a los proveedores privados con miras a promover sus propios objetivos. En un país centroamericano, El Salvador, una nueva reforma configuró un modelo diferente basado en el sector privado, que encaró algunos riesgos para ampliar los servicios de intermediación. El modelo salvadoreño es único en la región, porque propicia un servicio nacional brindado exclusivamente por proveedores privados o sin fines de lucro. Representantes del Ministerio de Trabajo y Previsión Social y de una institución estatal tripartita de capacitación —el Instituto Salvadoreño de Formación Profesional (INSAFORP) - tuvieron una importante función directriz en la formulación y el respaldo del nuevo servicio experimental e integrarán un comité supervisor de sus políticas, aunque sin competencia en su administración cotidiana. El experimento salvadoreño configura un servicio de intermediación laboral administrado enteramente por entidades privadas. Existen asimismo planes para la creación de una red de "asociados satélites" del servicio privado nacional de empleo. Diferente del caso salvadoreño es el de los países en los que sólo hay proveedores privados de intermediación laboral, y no existe un servicio público ni esfuerzos públicos y privados para colaborar en un sistema nacional. En estos casos el enfoque sería de laissez-faire: los proveedores privados pueden competir entre sí, pero no se hace esfuerzo alguno por defender o concretar el componente de "bien público" de los servicios de intermediación ni por coordinar el 
mercado laboral y las políticas de capacitación. La base teórica para un modelo puramente privado radica en el argumento de que no existe una función de bien público en la intermediación, y por lo tanto cualquier servicio público de empleo o subsidio público a los proveedores privados acarrearía ineficiencias en el mercado.

La gran variedad de modelos de intermediación descritos demuestra de manera elocuente que no existe una única forma correcta de estructurar los servicios de intermediación laboral. Cuál de los modelos resulte más eficaz, tenga mejores resultados y atienda a la mayor cantidad y variedad de usuarios, dependerá del contexto del país, la fuerza y el desempeño de las instituciones públicas y privadas, la financiación disponible y los vínculos entre los actores sociales clave. Las instituciones y su estructura influyen ciertamente en la prestación eficaz de los servicios de intermediación laboral, y en las personas que la reciben. En un estudio realizado recientemente en la OCDE se observa que la estructura institucional [de los servicios de empleo] efectivamente incide en factores como el conjunto de conocimientos y experiencia acumulada que contribuye a la gestión, la disposición de las partes involucradas a cooperar en la puesta en práctica de las estrategias concretas y la percepción de que su funcionamiento tiene legitimidad política (OCDE, 2000). La financiación pública específicamente dirigida a compensar el costo más alto de la colocación de los trabajadores menos favorecidos (por ejemplo, en las variantes de los modelos C, D, E y posiblemente el F) parece ser la herramienta más eficaz para evitar la discriminación que resultaría de una selección de los destinatarios de los servicios únicamente sobre la base de las fuerzas del mercado. La sola financiación pública del servicio, sin mecanismos concretos de focalización en determinados grupos, ha demostrado su ineficacia para proteger adecuadamente a los trabajadores de menores ingresos y menos favorecidos.

\section{VI}

\section{Consideraciones y recomendaciones para los países de América Latina y el Caribe}

\begin{abstract}
Al introducir nuevos sistemas de intermediación laboral o reestructurar los existentes, la región enfrenta particulares desafíos y necesidades económicas que les son propias. Será de gran utilidad identificar las condiciones económicas específicas para invertir en reformas de los sistemas de intermediación laboral y considerar de qué manera esos sistemas deberán adaptarse a las características de las economías de América Latina y el Caribe.

Las principales motivaciones económicas de los sistemas de intermediación laboral son las mismas en los países desarrollados y en aquellos en desarrollo: en unos y otros la información sobre el mercado laboral es incompleta, hay ineficiencias en la intermediación de la demanda y la oferta de empleo y capacitación, y esto se ha vuelto más costoso en un mercado de trabajo globalizado y en rápida transformación. En el Ministerio del Trabajo y Promoción Social de Perú se llegó a la conclusión de que la escasa información sobre el mercado laboral y una intermediación ineficaz se traducían en tasas de desempleo más elevadas, importantes desajustes ocupacionales y subutilización
\end{abstract}

de la fuerza de trabajo, todo lo cual disminuía el bienestar social y justificaba la necesidad de reformar el servicio de intermediación laboral de la nación.

En todos los países, la función de colocación, que es el cometido central de los servicios de intermediación laboral, se realiza de manera más eficaz en un contexto de expansión económica. Sin embargo, hay condiciones de crecimiento limitado en las que sigue siendo razonable invertir en sistemas de intermediación laboral. Esto sucede básicamente cuando en los mercados laborales se perciben tendencias que pueden ser subsanadas en gran medida con una mejor intermediación. Entre esas tendencias se hallan una alta rotación laboral, elevado desempleo de corto plazo, grave discriminación o falta de transparencia en el mercado laboral, y fallas sistemáticas en capacitación y empleo, fenómenos todos fáciles de encontrar en los mercados laborales de América Latina y el Caribe, y que merecen ser evaluados como importantes campos para la acción de los sistemas de intermediación laboral. Sin embargo, si un país enfrenta una auténtica crisis de desempleo estructural alto (el caso de Argentina), 
las inversiones en los servicios de intermediación laboral no deberían ser prioritarias. En situaciones de crisis la eficacia de la intermediación se verá muy limitada, salvo que el servicio también coordine o aplique programas de compensación de ingresos u otros característicos de las redes de seguridad social.

Una tendencia económica que es clave en los sistemas de intermediación de la OCDE no resulta tan trascendente para América Latina y el Caribe. Los sistemas de intermediación de la OCDE han estado focalizándose crecientemente en el problema más enquistado en sus mercados laborales: el desempleo de largo plazo. Irónicamente, los servicios de intermediación no se concibieron en modo alguno para abordar este problema, sino con el objeto de reducir el desempleo transitorio, de corto plazo, y mejorar la coordinación de trabajadores y puestos.

Vemos así que, en general, las razones para reformar o fortalecer los sistemas de intermediación laboral en América Latina y el Caribe son sólidas, salvo en circunstancias de contracción seria del empleo. Y lo son aún más en los países de la región con cifras altas de desempleo de corto plazo, vínculos débiles entre los sistemas de capacitación y empleo y niveles altos de discriminación. Sin embargo, los beneficios de los servicios de intermediación no se perciben con rapidez, por lo que las expectativas de que ellos mejoren en la región el desempeño en el mercado laboral de grupos con necesidades especiales de empleo deberían ser moderadas.

Ciertamente la forma en que cada país aborde las reformas o medidas de fortalecimiento dependerá de su situación y del estado de sus servicios de intermediación laboral. La intermediación laboral debería considerarse como un instrumento de política, integrado a un sistema más amplio de empleo y capacitación adaptado a las necesidades e instituciones nacionales. Un elemento decisivo en toda reforma o fortalecimiento de los servicios de intermediación laboral es la visión de largo plazo de las metas del servicio y de la correspondiente estructura institucional. En general, puede decirse que la reforma y el fortalecimiento de los servicios de intermediación funcionan a un doble nivel, uno más potente que el otro (cuadro 3). El primer nivel, más modesto, corresponde a la aplicación de medidas para fortalecer y hacer más eficaz el servicio nacional de intermediación, sin alterar su estructura institucional básica. Abarca, entre otros aspectos, la capacitación de personal, el mejoramiento de la gestión, la expansión de los servicios y el establecimiento de servicios nuevos. El segundo nivel combina el mejoramiento de la gestión y eficiencia del servicio con la reforma y la reestructuración institucionales. La experiencia internacional ha mostrado claramente que mediante la reforma y reestructuración institucionales se obtienen beneficios mayores. A través de la reestructuración se establecen nuevas condiciones de mercado con los sectores privado y sin fines de lucro, posibilitando avances de mayor entidad en la cobertura y la eficacia. En Perú y El Salvador, esto significó replantear el papel desempeñado por los proveedores públicos, privados y sin fines de lucro en un sistema nacional de intermediación, considerando las fortalezas y debilidades de las instituciones nacionales y la mejor manera de ampliar el uso del sistema por los empleadores locales. El establecimiento de redes y asociaciones puede iniciarse sin que haya habido una reestructuración sustancial de las instituciones existentes, $\mathrm{o}$ antes de efectuar una reestructuración completa. El cuadro 3 presenta un resumen de las principales medidas que deberán tomarse para el fortalecimiento (nivel 1) y la reforma (nivel 2) de los sistemas de intermediación laboral.

La reestructuración y la reforma institucionales pueden tener particular importancia en los países de América Latina y el Caribe, donde la eficacia de los sistemas actuales de intermediación y su credibilidad en el medio empresarial es tan limitada. Dadas las restricciones presupuestarias en toda la región, no es fácil concebir un servicio exclusivamente público que cuente con financiamiento suficiente para funcionar sin asociarse a agentes del sector privado y sin fines de lucro.

A la hora de crear nuevos marcos institucionales y modernizar los servicios de intermediación, los países de América Latina y el Caribe deberán tener en cuenta las características distintivas de sus mercados laborales para configurar sistemas que se adapten mejor a sus realidades. Estas características, propias de las economías en desarrollo, no invalidan las razones a favor de la reforma y el fortalecimiento de los sistemas de intermediación laboral, pero sí influyen en el tipo de servicios de intermediación y de servicios específicos necesarios, y exigen la configuración de un modelo de intermediación más adecuado para América Latina y el Caribe. A continuación se examinarán tres aspectos clave.

\section{El papel del sector informal}

Los sistemas de intermediación laboral han hecho hincapié tradicionalmente en el empleo en el sector 


\section{Nivel}

Nivel 1:

Medidas de fortalecimiento y previas a las reformas
Ejemplos de medidas de política

Propiciar un marco legal apropiado para ampliar los proveedores privados y sin fines de lucro, incluso con cambios en la legislación y reglamentación

Crear redes experimentales con proveedores privados y con aquellos sin fines de lucro

Mejorar la capacitación del personal

Perfeccionar los sistemas informáticos, computarizar los "bancos de puestos" y modernizar los equipos

Fortalecer el aporte y la participación de los empleadores

Introducir reformas de gestión y medidas de desempeño

Poner en marcha servicios especializados, concebidos a medida (por ejemplo, evaluación de competencias, servicios a los empleadores)

Realizar campañas para promover el uso de los servicios
Nivel 2:

Reforma o reestructuración institucional
Reestructurar el marco y el modelo institucionales (por ejemplo, modificar los roles de los sectores gubernamental, privado y sin fines de lucro)

Ampliar el mandato del servicio y su política de coordinación del mercado laboral para abarcar, entre otras cosas, la suma de nuevas funciones clave como los vínculos con el sistema de capacitación, los servicios de empleo por cuenta propia y la provisión de servicios sociales

Establecer sistemas de información que integren datos sobre los que buscan trabajo, los puestos disponibles y el mercado laboral. formal, ya que respecto de este sector los empleadores se muestran más dispuestos a anunciar vacantes y a solicitar personal. Sin embargo, la realidad en muchos países de América Latina y el Caribe indica que el sector informal y de trabajo por cuenta propia puede ser la fuente de empleo más significativa, sobre todo en áreas rurales y para los trabajadores de mayor edad. En la oficina del servicio nacional de empleo en Brasilia se comprobó que solamente un $2 \%$ a $3 \%$ de los trabajadores de más de 33 años que había perdido un puesto en el sector formal volvía a encontrar empleo en ese sector. ${ }^{3}$ Existen pocos estudios empíricos del uso de los sistemas de intermediación laboral en el sector informal, o del efecto que pueden tener sobre el aumento o la disminución de la informalidad. Resulta evidente que en países en los que la tasa de informalidad es elevada y se acompaña de altas cifras de subempleo, el mejoramiento de la coordinación entre los trabajadores y los empleos en el sector formal, y el fomento de las condiciones conducentes a la formalizar la creación de pequeñas empresas, arrojará resultados positivos. Un punto clave para los sistemas de intermediación de la región será la ampliación del concepto de empleo a la realidad, para abarcar el envío de personas a programas de

\footnotetext{
${ }^{3}$ Entrevista con Ivan Ribeiro Guimarães Gonçalves, realizada por la autora el 14 de mayo de 1998.
}

microempresas, de empleo por cuenta propia y de desarrollo de negocios a pequeña escala, dado que ellos son fuente habitual de empleo - y a veces la únicapara una porción de la fuerza laboral. Hay proveedores nacionales (por ejemplo, instituciones gubernamentales y organizaciones no gubernamentales) que ofrecen programas de empleo por cuenta propia y para la microempresa, pero no a cualquiera que lo desee solicitar. Mucho menos frecuentes aún son los centros de información que brindan datos sobre programas alternativos y asesoran a los emprendedores potenciales en la elección del que más se ajuste a sus necesidades.

Los sistemas de intermediación laboral de la región con altos niveles de informalidad podrían considerar la posibilidad de brindar asesoramiento e información en dos áreas: la formalización de empresas y el envío de los usuarios a los servicios pertinentes. Esto difiere mucho de la función reguladora de las condiciones sanitarias y de seguridad de las empresas y de su cumplimiento de las normas laborales y comerciales. Una de las claras lecciones extraídas hasta el momento es que la función reguladora es una de las que menos se adapta a las competencias de un centro de intermediación laboral. Lo que se pone de relieve en este artículo es la importancia de proporcionar información voluntariamente, sobre todo cuando desde el gobierno se ofrecen procedimientos acelerados para el registro de las empresas. Las dos recomendaciones 
para este tipo de servicios tienen en cuenta una enseñanza derivada de las ventanillas únicas (los one-stop shops de los países industrializados); esto es, el reconocimiento cada vez mayor, por parte de los servicios de intermediación, de que los límites del concepto de empleo se han vuelto menos nítidos en lo concerniente a la búsqueda de trabajo como empleado o empleador, y que los servicios de intermediación deben seguir vigentes allí donde existen empleos.

\section{Grupos excluidos del mercado laboral}

Los mercados de trabajo en los países de América Latina y el Caribe también encaran grandes problemas debidos a que la poca calificación, la pobreza y la discriminación laboral se concentran en grupos específicos, que pueden abarcar, entre otros, a las mujeres pobres, las poblaciones indígenas, las personas con discapacidades, los portadores del virus de inmunodeficiencia humana o los enfermos con sida. En años recientes, en la región se ha comenzado a comprender que la exclusión de ciertos grupos de todas las oportunidades sociales y económicas explica en parte la alta tasa de desigualdad y pobreza que persiste en sus países.

Entre los usos potenciales de los sistemas de intermediación laboral se cuenta el de atender a estos grupos de manera más eficaz, mediante servicios especializados y actividades de divulgación. Sin embargo, como sucede con la focalización en los desempleados de largo plazo en la OCDE, a estos grupos se accede con más facilidad a través de la gestión de casos individuales combinada con servicios especializados y el envío a servicios sociales. El procedimiento empleado en el Reino Unido resulta particularmente interesante: con el fin de atender las necesidades de las minorías étnicas y los grupos menos favorecidos, el servicio nacional contrata a organizaciones no gubernamentales especializadas para que entreguen los servicios.

El desafío que enfrentan los servicios de intermediación laboral en América Latina y el Caribe es el de aumentar la cobertura de las personas menos favorecidas junto con ampliar la base general de usuarios y empleadores, para crear más oportunidades de que las personas menos favorecidas accedan a trabajos de mejor calidad.

\section{Trabajo migratorio y estacional}

Inevitablemente, en los mercados laborales de América Latina y el Caribe se registra una importante emi- gración tanto hacia los Estados Unidos y Europa como hacia otros países de la región. En la República Dominicana y El Salvador, por ejemplo, las remesas de dinero que efectúan los trabajadores de esos países radicados en el extranjero son una fuente esencial de los ingresos nacionales y distorsionan considerablemente el funcionamiento del mercado laboral local. En los países caribeños cuya principal actividad es el turismo, las oportunidades laborales se reducen en la temporada baja, con la consiguiente emigración. Cabe preguntarse, y es debatible, si un servicio nacional de intermediación laboral debería desempeñar algún papel en la regulación de la migración temporal o estacional, o en la protección y supervisión de los trabajadores que migran dentro de la región o fuera de ella. En los países que han optado por servicios de emigración, se argumenta que así se les brinda a sus trabajadores una modalidad legal y segura de emigración y se les garantiza el regreso sin riesgos a su país de origen. En México, por ejemplo, el servicio de empleo público, en virtud de un acuerdo con el gobierno canadiense, selecciona un conjunto de trabajadores agrícolas a los que se otorgan visas legales y paga el costo de su traslado, para que se empleen de manera estacional en Canadá. El servicio de empleo nacional de Barbados, como parte de programas especiales, lleva registros de las oportunidades de empleo estacional fuera del país, sobre todo en Canadá y en cruceros estadounidenses. La función principal y dominante de cualquier servicio de intermediación es fomentar el mejoramiento de los mercados laborales del país.

La decisión de establecer programas especiales para la migración estacional o temporal, y la forma de hacerlo, competen a cada país y dependen de las circunstancias nacionales. En ningún país de la región el servicio de empleo se dedica principalmente a las colocaciones en el extranjero, como sucede en Filipinas o Sri Lanka, ni sería conveniente que lo hiciera. En Panamá no se permiten las agencias privadas especializadas en el empleo en el extranjero. Pero la respuesta y la adecuación a las tendencias migratorias son problemas de carácter urgente para los dirigentes regionales. Los directores de los servicios de empleo centroamericanos, por ejemplo, defienden la importancia de seguir enfrentando la realidad de una considerable migración internacional mediante encuentros periódicos que permitan compartir información sobre experiencias nacionales, proponer políticas nacionales y encontrar instrumentos para garantizar condiciones de trabajo adecuadas a los migrantes que atraviesan las fronteras nacionales. 


\section{VII}

\section{Hacia un modelo de intermediación laboral propio de América Latina y el Caribe}

Aunque de la evolución de los sistemas de intermediación laboral en los países de la OCDE sea posible extraer numerosas conclusiones válidas para América Latina y el Caribe, habrá aspectos en los que se deberá encontrar soluciones originales, de manera que los sistemas de intermediación se adapten lo mejor posible a las necesidades de la región y a las fortalezas y debilidades de sus instituciones. Los países en desarrollo tienen mucho menos experiencia que la OCDE en sistemas de intermediación laboral y en muchos casos han soportado restricciones derivadas de presupuestos limitados, instituciones débiles y sectores privados de desarrollo lento. El activismo del sector sin fines de lucro y su capacidad de llegar a las poblaciones marginadas de América Latina y el Caribe es una carta que no se debería dejar de lado en la construcción de nuevos sistemas de intermediación laboral. Los sindicatos también son nuevos espacios para la cooperación en la intermediación. A riesgo de generalizar demasiado, a continuación se enumeran algunas medidas que podrían considerarse para futuros sistemas en la región:

- Establecer una red de proveedores de servicios de intermediación laboral públicos, privados y sin fines de lucro y probar nuevos marcos institucionales que logren la confianza y participación de los empleadores privados.

- Allí donde el empleo en el sector informal es alto, incorporar tanto el trabajo asalariado como aquel por cuenta propia al sistema de intermediación laboral, mediante el establecimiento de un centro de información y de remisión a programas de trabajo por cuenta propia. Esto daría origen a centros de intermediación que, como mínimo, refle- jarían la realidad del empleo en el país, sin distorsionarla.

- Incorporar el suministro de información sobre capacitación y remitir a las personas a las instituciones pertinentes de manera que los servicios de intermediación fomenten un vínculo productivo entre la capacitación y el empleo. No es recomendable que los servicios de intermediación laboral de la región se vuelvan proveedores de capacitación. En la OCDE ha quedado demostrada la importancia de separar las funciones de contratante y de proveedor de la capacitación. Sin embargo, la existencia de un rol "intermediario" entre la capacitación y el empleo es claramente necesaria en la región.

- Dar especial atención a los grupos menos favorecidos, a través de servicios especializados y estrategias focalizadas.

- Desacelerar la evolución de los sistemas de autoservicio computarizados como los de la OCDE hasta que el uso de las tecnologías de la información esté más difundido en el país.

- Eliminar las funciones regulatorias de los servicios de intermediación.

- Incorporar los datos sobre intermediación laboral en los análisis más amplios de mercados laborales a nivel nacional y regional.

- A medida que evolucionan los sistemas nacionales, desarrollar paralelamente subsistemas regionales de capacitación e intermediación laboral (por ejemplo, en toda Centroamérica) que respalden las tendencias de los mercados laborales y la integración regional.

(Traducido del inglés) 


\section{Bibliografía}

Dockery, A. M. y T. Stromback (2001): Devolving public employment Services: preliminary assessment of the Australian experiment, International Labour Review, vol. 140, $N^{\circ} 4$, Ginebra, Organización Internacional del Trabajo (OIT).

Fay, R. G. (1997): Making the Public Employment Service More Effective Through the Introduction of Market Signals, Labour Market and Social Policy Occasional Papers, No 25, París, Organización de Cooperación y Desarrollo Económicos (OCDE).

Flores Lima, R. (1999): A new state of decentralization in Mexico: granting autonomy to the states, Decentralizing Employment Policy: New Trends and Challenges. The Venice Conference, París, Organización de Cooperación y Desarrollo Económi$\cos (\mathrm{OCDE})$.

Fretwell, D. y S. Goldberg (1993): Developing Effective Employment Services, World Bank Discussion Papers, No 208, Washington, D.C., Banco Mundial.

Martin, J. P. (1998): What Works Among Active Labour Market Policies: Evidence from OECD Countries' Experiences, París, Organización de Cooperación y Desarrollo Económicos (OCDE).

Martínez Espinoza, E. (1997), Chile: Experiences in a Marketoriented Training System, Ginebra, Organización Internacional del Trabajo (OIT), diciembre.

Mazza, J. (1999): Unemployment Insurance: Case Studies and Lessons for Latin America and the Caribbean, Working Paper Series, $N^{\circ} 411$, Washington, D.C., Banco Interamericano de Desarrollo (BID), octubre.
Ministerio de Trabajo y Promoción Social (2001): La modernización del Servicio Nacional de Empleo en el Perú: una propuesta aplicable a países en desarrollo, documento preparado para el quinto Congreso Mundial de la Asociación Mundial de Servicios Públicos de Empleo.

OCDE (Organización de Cooperación y Desarrollo Económicos) (1997): Labour Market Policies: New Challenges. Enhancing the Effectiveness of Active Labour Market Policies: A Streamlined Public Employment Service, París.

(2001), Labour Market Policies and the Public Employment Service, París.

(2000): The Public Employment Service in the United States, París, julio.

(1998): Documento de conclusiones del Seminario-Taller sobre la estructura y funcionamiento de los departamentos de empleo y de los sistemas de intermediación en el mercado de trabajo (San Rafael de Heredia, Costa Rica, 8 y 9 de septiembre de 1998).

OIT (Organización Internacional del Trabajo) (1999): Documento de conclusiones del Segundo Seminario-taller sobre la estructura y funcionamiento de los departamentos de empleo y de los sistemas de intermediación en el mercado de trabajo (San Salvador, 12 y 13 de mayo de 1999).

Thuy, Ph., E. Hansen y D. Price (2001), The Public Employment Service in a Changing Labour Market, Ginebra, Oficina Internacional del Trabajo. 
